\section{ASYMMETRIC FLOW VELOCITY MEASUREMENT USING MULTIPATH ULTRASONIC FLOWMETER WITH ADAPTIVE WEIGHTING METHOD GUIDED BY TOMOGRAPHY}

Khairul Amri, Suprijanto*, Deddy Kurniadi, Endang Juliastuti

Instrumentation and Control Research Group, Institut Teknologi Bandung, Indonesia, Labtek VI, 2th Floor Ganesha 10 Bandung Indonesia
Article history

Received

10 September 2019

Received in revised form

20 November 2019

Accepted

3 December 2019

Published online

25 December 2019

*Corresponding author

supri@tf.itb.ac.id

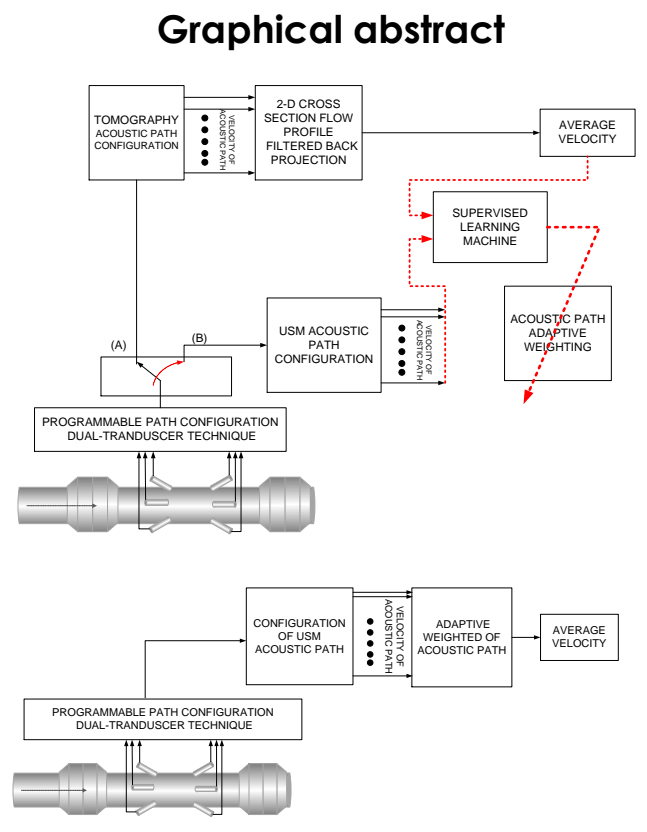

\begin{abstract}
A conventional transit time ultrasonic flowmeter (USM) has a high accuracy for symmetric flow profiles but inaccurate for asymmetric flow profiles. Flow profile shapes can also change over time and difficult to predict. USM with tomographic configuration (USM-Tomo) can adapt to the flow profile changes but result in low temporal resolution. Meanwhile, USM with an adaptive weighting method can measure asymmetric flow velocity but limited to specific asymmetric flow profiles. An alternative scheme to determine adaptive weighting in various asymmetric flow profiles, we proposed a hybrid USM-Tomo. This scheme proposes programmable acoustic path configuration that could set the path mode between USM and tomography. Reducing computation of time of flight in each acoustic can be done by applying the dual-transducers technique. An adaptive weighting of hybrid USM-Tomo is calculated based on the mapping function between the set of velocity on 6 parallel paths of USM and average flow velocity from USMTomo. The mapping function is determined using machine learning, i.e., Artificial Neural Network (ANN) and Support Vector Regression (SVR). In the measurement phase, the average flow velocity is determined using the mapping function with input 6 parallel acoustic paths. Based on various types of asymmetric flow profiles used in the experiment, the 6 parallel acoustic paths of USM could produce average flow velocity with error below $1 \%$ compared to USM-Tomo. Therefore, the proposed hybrid USM-Tomo scheme has potential to be an alternative scheme for flow meter in industrial application.
\end{abstract}

Keywords: Ultrasonic flowmeter, transit time method, dual-transducers, tomography, Hybrid USM-Tomo, Artificial Neural Network, Support Vector Regression

(C) 2020 Penerbit UTM Press. All rights reserved

\subsection{INTRODUCTION}

A transit time ultrasonic flowmeter (USM) has some advantages; i.e., high accuracy, no moving parts, no pressure drop, and suitable for gases and liquids. Currently, it is used in many applications such as mining [1], cryogenic [2], custody transfer [3], hydrogen flow [4] and intake turbine power plan [5]. 
To maintain USM accuracy, AGA-9 recommended installation guidance for USM's practitioners [6]. A flow conditioner is used to convert asymmetric into symmetric flow with high repeatability [7], [8]. In this condition, the average flow velocity on the crosssection pipe was calculated by integrating the local velocity along all fixed weighted acoustic paths. The basic scheme of conventional USM is shown in Figure 1 , where it is assumed that the flow profile is symmetric or fully developed flow.

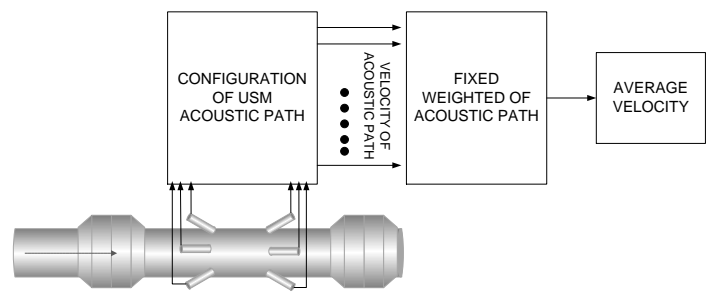

Figure 1 Conventional USM

However, the flow conditioner can cause pressure drop [8], and the problems get worse if it has a blockage, corrosion, surface wear, and emergence of the metal deposition [9]. Without flow conditioning, the USM needs to be installed at least 50D $(D=$ diameter) from a straight run upstream flow [6]. Thus, it is often difficult in practice due to a lack of space. If AGA-9 guidance was not fully implemented, the flow profiles might become highly asymmetric, and the application of a conventional USM becomes inaccurate. Besides the installation condition [3], [10], [11], [12], [13], a combination of many factors may produce asymmetric profile such as irregular pump performance, the roughness of pipe's inner surface, and transducer installation [14], [15], [16]. Those factors are hard to eliminate. Therefore, many researchers have proposed several advance USM schemes to maintain accuracy in spite of asymmetric profiles presence.

The first key to advance USM schemes development is an optimal configuration of the acoustic path and an optimal number of parallel paths. Theoretically, if the number of coverage paths was increased, the better the accuracy of the average flow velocity has the potential to be achieved [17]. Performance evaluation of different forms and the number of USM configuration are also analyzed and compared [12], [13], [18], [19]. The number of parallel paths configurations from 4 to 18 was investigated [5], [12], [20]. More complex path configurations, such as 3D isoscale [17], a star with 36 paths [21] are also reported. The second key to advance the development of USM schemes is the determination of the set of adaptive weighting in each specific path which is a key and a crucial step to maintaining the accuracy of average flow velocity on asymmetric flow profiles. The general scheme of advance USM is shown in Figure 2 for the training phase, and Figure 3 for the measurement phase.

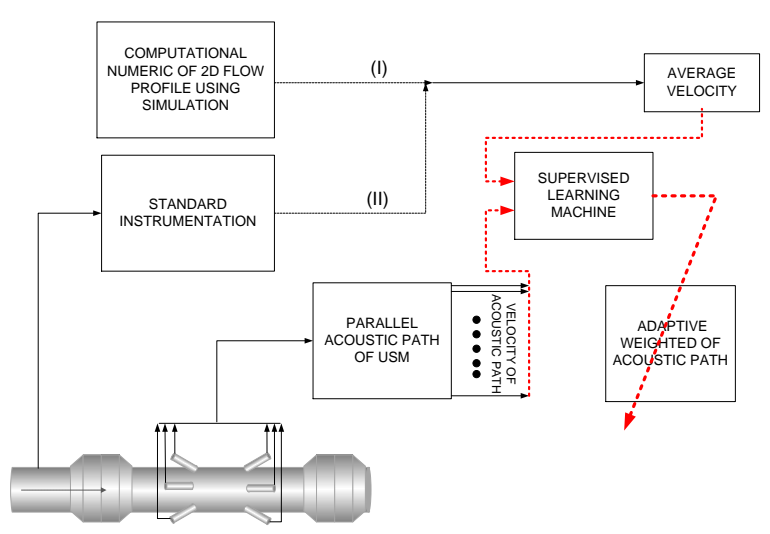

Figure 2 The general scheme of advance USM in training phase. (Note (I): training set is obtained from simulation and (II): target training is average velocity using standard instrumentation)

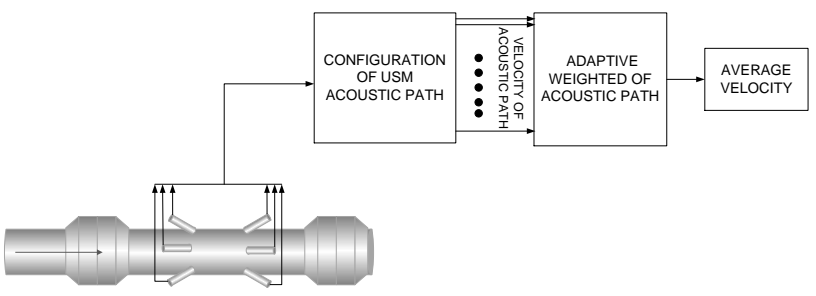

Figure 3 The general scheme of advance USM in measurement phase

A proper adaptive weighted of the acoustic path must be determined in the training phase. Previous researches have been investigated the several methods for supervised learning machine, such as support vector regression and machine [10], [25]. The other method is based on artificial neural networks (ANN), and some new processes to optimization weighting, such as genetic algorithm, extreme learning have been reported [22], [23], [24], [38]. The scheme of advance USM that reported on [10], [22], [23], [24], [25], [38], on the training phase and also on the measurement phase are only using data that generated by computational numeric of $2 \mathrm{D}$ flow profile simulation.

Zheng [26] proposed the other approach that in the training phase using datasets from simulation data. Then the obtained weighting is used to determine the average flow velocity on the measurement phase. Disadvantage of the Zheng scheme is hard to cover a typical flow profile in a real measurement flow [3]. The other approach is proposed by Tang et al. [27]. In this approach, a flow data that measured using a conventional flow meter (i.e. hot wire anemometer, turbine meter) is used datasets in the training phase.

Using Tang et al. approach, the weighting that used to build the mapping function is limited. Because in the real situation, the change of asymmetric profile shape cannot be predicted far in advance, such as 
due to sediments on the pipe wall and pipeline installation.

This paper proposed a better approach as an alternative scheme for determining adaptive weighting in the guided training phase of USM-Tomo, named a hybrid USM-Tomo. Computation of time of flight in each acoustic path attempt to increase by applying the dual-transducers technique. Its technique applied two pairs of transmitter-receiver transducers that could determine a time of flight simultaneously. In this proposed scheme, an adaptive weighting was represented as a mapping function between flow velocity in each path and a target of average velocity in various possible patterns of flow profiles. In our preliminary research, the type of ANN architecture and learning methods for support advance USM in asymmetry flow velocity have been investigated [38]. The potential type of ANN architecture and learning methods for multipath USM has been evaluated. The performance of ANN to determine the desired adaptive weighted of acoustic path has been tested only on 2D flow profile simulation. In this work, further investigation method of a supervised learning method was done. The performance of ANN and Support Vector Machine (SVM) is co-evaluated and compared to determine the optimal mapping function of the acoustic path on the 2D asymmetric flow profile on simulation and the experiments.

\subsection{METHODOLOGY}

\subsection{Multipath USM with Transit Time}

The transit time USM can be composed of single or multipath. Every path is formed by a pair of transducers, upstream (Tu) and downstream (TD) transducer, as seen in Figure 4. During the measurement process, the transit time of ultrasonic waves from Tu to Td ( $\left.t_{u}\right)$ and reversely from Td to To ( $\left.t_{D}\right)$ are both recorded. The average flow velocity (Vpath_1) for the single path can be calculated by using Eq. 1 [18], [19]. D is the diameter of the pipe, and $\boldsymbol{\theta}$ is the path angle.

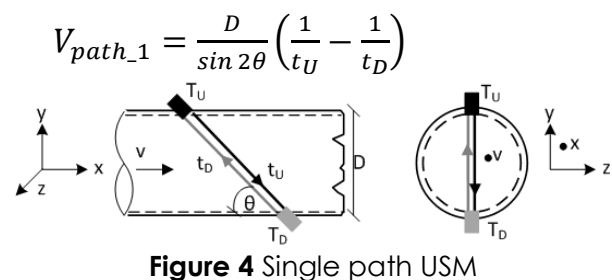

The most conventional USM uses a pair of transducers working as both transmitter and receiver interchangeably, so tu and to were obtained sequentially. The total time for each acoustic path (tcon) is

$$
t_{\text {con }}=t_{U}+t_{D}
$$

In the preliminary experiment [30], reducing the time of $t_{\text {con }}$ can be achieved using two pairs of transmitterreceiver transducers on each path. Therefore, tu and to can be recorded at the same time, namely dualtransducers techniques. The example of multipath configurations using dual-transducers viewed in the cross-section pipe was shown in Figure 5.

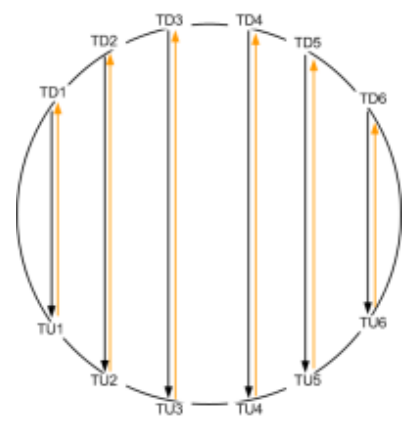

Figure 5 Illustration of multipath with parallel configuration using 6 parallel paths with dual-transducers techniques. tu and $t_{d}$ on each path was recorded at the same time

In common USM, a multipath configuration is often used. The average of flow velocity in cross-section pipe $\left(V_{\text {avr }}\right)$ is calculated by integrating the local velocity along all measurement paths (see Figure 5), $\vee_{\text {avr }}$ can be determined by

$$
V_{a v r}=K_{h} \sum_{n=1}^{i} w_{n} V_{p a t h-n}
$$

where a fixed weighted acoustic path $\left(\mathbf{w}_{\mathbf{n}}\right)$ was defined as the contribution of $\mathbf{n}^{\text {th }}$ path to the average velocity is the number of paths, $\mathbf{V}_{\text {path-I }}$ denotes the velocity the $\mathbf{n}^{\text {th }}$ path and $\mathbf{K}_{\mathbf{h}}$ is the correction meter factor. As we mention above, $\mathbf{V}_{\text {avr }}$ using Eq 3 , is limited for symmetric flow profile.

For asymmetric flow profile, adaptive weighting can be viewed as a mapping function $\mathbf{F}($.$) between$ $\left\{\mathbf{V}_{\text {path-1 }}, \mathbf{V}_{\text {path-2,........, }} \mathbf{V}_{\text {path-n }}\right\}$ into target value of $\mathbf{V}_{\text {arr }}$, that performed on training phase (See Figure 2). The relation between $\mathbf{V}_{\text {path-n }}$ and $\mathbf{V}_{\text {avr }}$ was modeled as

$$
V_{a v r}=F\left(w_{i k}, V_{p a t h-1}, V_{p a t h-2}, \ldots \ldots, V_{\text {path-n, }}\right)
$$

$\mathbf{F}($.) was determined by supervised learning. Therefore, $\mathbf{W}_{\mathrm{ik}}$ is depend on architecture machine learning that applied on training phase. Note, Eq. 4 is modelled for one type of asymmetric profile, where $\mathbf{V}_{\text {avr }}$ can be viewed as target value during the training phase.

\subsection{Scheme of Hybrid USM-Tomo}

It is assumed, on specific piping configurations such as setting of valves opening, blower speed, type of elbow and metering location, is related to the flow profile that possibly occurs. A set of flow profile patterns is generating using these assumptions on the training phase. Hybrid USM-Tomo is proposed to 
optimally determine a mapping function on the same multipath USM transit time.

The main difference between hybrid USM-Tomo with previous scheme that represented in Figure 2 is the programmable acoustic path that designed to work in tomography and USM configuration path using a specific multiplexing technique. The hybrid USM-Tomo scheme can be seen in Figure 6.

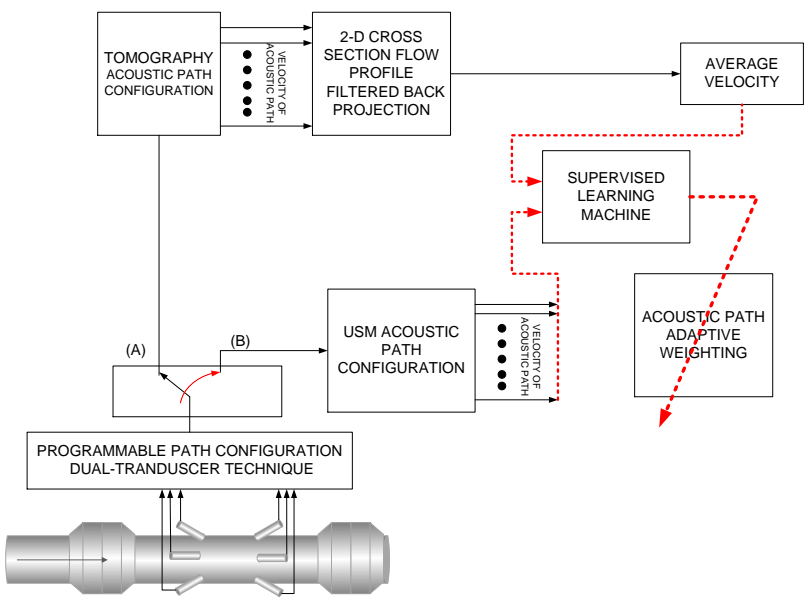

Figure 6 Scheme of hybrid USM-Tomo on training phase. (Note (A): path configuration for tomography and (B): path configuration for USM)

\subsubsection{USM Tomography}

Acoustic path of USM-Tomo must be configured to cover the entire cross-section pipe [18], [28]. In USMTomo, the object is the flow profile $\left(\mathbf{V}_{\text {томо }}(\mathbf{x}, \mathbf{y})\right)$ while the projection data $\left(\mathrm{Pe}_{\boldsymbol{\theta}}(\mathbf{t})\right)$ at particular angle is obtained from the parallel path of USM-Tomo that have been grouped according to its orientation (see Figure 7).

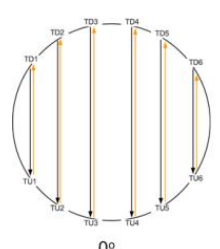

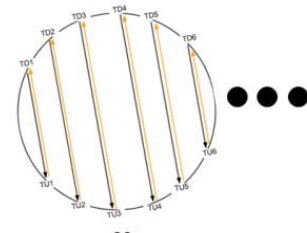

$30^{\circ}$

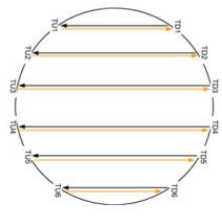

150
Figure 7 Scheme of acoustic path configuration on USMTomo. Rotation of standard parallel path is used to cover the entire cross-section pipe

In a specific angle $\mathbf{q}$, set of $\left\{\mathbf{V}_{\text {path-1 }}, \mathbf{V}_{\text {path-2, }}, \ldots, \mathbf{V}_{\text {path- }}\right.$ n\} defined as the projection data of the object at angle $\boldsymbol{\theta}\left(\mathbf{P}_{\boldsymbol{\theta}(\mathrm{t})}\right)$. Set of $\boldsymbol{P}_{\boldsymbol{\theta}(\mathrm{t})}$ on the entire cross-section pipe can be viewed as a slice. The $2 \mathrm{D}$ reconstruction of velocity profile $\mathbf{V}_{\text {томо }}(\mathbf{x}, \mathrm{y})$ use the Fourier Slice Theorem (FST). The FST states that ID Fourier transform of the projection data of the object at angle $\boldsymbol{\theta}\left(\mathbf{P}_{\boldsymbol{\theta}(\mathrm{t})}\right)$ is equal to $2 \mathrm{D}$ Fourier transform $\left(\mathbf{S}_{\boldsymbol{\theta}}(\mathbf{w})\right)$ of the object slice at the same angle [31], represented as

$$
S_{\theta}(w)=\int_{-\infty}^{\infty} P_{\theta}(t) e^{-j 2 \pi w t} d t
$$

where,

$$
t=x \cos \theta+y \sin \theta
$$

Filtered back projection (FBP) used to reconstruct of $\mathrm{V}_{\text {томо }}(\mathbf{x}, \mathbf{y})$. The FBP starts with Fourier transforms on projection data $\mathbf{P}_{\boldsymbol{\theta}}$ as in Eq. (5). $\mathbf{S}_{\boldsymbol{\theta}}$ is then filtered before the inverse Fourier is used to obtain $\mathbf{Q}$ (Eq. (6)). The filter functions $(\mathbf{w})$ are employed to give weight to each projection in the frequency domain.

$$
Q_{\theta}(w)=\int_{-\infty}^{\infty} S_{\theta}(w)|w| e^{j 2 \pi w t} d w
$$

The velocity at each data point is calculated using Eq. (7). $\mathbf{k}$ is the number of projections, $\mathbf{Q}_{\boldsymbol{\theta}}$ is the filtered projection, $\boldsymbol{\theta}$ denotes the projection angle, $\mathbf{x}$ and $\mathbf{y}$ are the coordinate respected to the velocity point. Finally, the average of $\mathbf{V}_{\text {томо }}(\mathbf{x}, \mathbf{y})$ is computed using the average method, as written in Eq. (8).

$$
\begin{gathered}
V_{\text {томо }}(x, y)=\frac{\pi}{K} \sum_{i=1}^{K} Q_{\theta_{i}}\left(x \cos \theta_{i}+y \sin \theta_{i}\right) \\
V_{\text {томо-AVR }}=\frac{1}{n m} \sum_{x=1}^{n} \sum_{y=1}^{m} V_{\text {Tомо }}(x, y)
\end{gathered}
$$

where $\mathbf{n}$ and $\mathbf{m}$ is row and column in the matrix of $\mathrm{V}_{\text {томо }}(\mathrm{x}, \mathrm{Y})$.

\subsubsection{Supervised Learning Process on USM-Tomo}

On training phase, a mapping between a set of velocity on each acoustic path of USM configuration (input) and $\mathrm{V}_{\text {TOMO-AVR }}(\mathrm{X}, \mathrm{Y})$ (target) are performed using supervised learning machine.

Given $\mathbf{m}$ patterns of 2D asymmetric flow profile, then the target can be represented as $\left\{\mathbf{V}_{\text {Tomo-AVR- }}\right.$ $\left.1(\mathbf{x}, \mathbf{y}), \quad \mathbf{V}_{\text {TOMO-AVR-2 }}(\mathbf{x}, \mathbf{y}), \ldots . . . . . . . . ., \mathrm{V}_{\text {TOMO-AVR-m }}(\mathbf{x}, \mathbf{y})\right\}$. Using the scheme of hybrid USM-Tomo (see Figure 6), on a specific pattern of $2 \mathrm{D}$ asymmetric flow profile has a set of $\left\{\mathbf{V}^{1}\right.$ path-1, $\mathbf{V}^{1}$ path-2,......... $\left.\mathbf{1}_{\text {path-n }}\right\}$ in USM path configuration and $\mathbf{V}_{\text {TOMO-AVR-1 }}(\mathbf{x}, \mathbf{y})$. In general, a set of $\mathbf{V}_{\text {path-n }}$ and $\mathbf{V}_{\text {TOMO-AVR-m }}(\mathbf{x}, \mathbf{y})$ can be represented as Eq. 9

Set of data input-target is generated for each specific flow profile that possibly occur on measurement phase. In this work, machine learning methods based on artificial neural network (ANN) and Support Vector Machine (SVM) [10], [25], [22], [32] are evaluation to generate optimal acoustic paths weighting.

$$
\text { input }=\left\{\left[\begin{array}{c}
V_{\text {path-1 }}^{1} \\
V_{\text {path-2 }}^{1} \\
\cdot \\
\cdot \\
V_{\text {path } n}^{1}
\end{array}\right],\left[\begin{array}{c}
V_{\text {path-1 }}^{2} \\
V_{\text {path-2 }}^{2} \\
\cdot \\
\cdot \\
V_{\text {path }-n}^{3}
\end{array}\right], \ldots .,\left[\begin{array}{c}
V_{\text {path }-1}^{m} \\
V_{\text {path-2 }}^{m} \\
\cdot \\
\cdot \\
V_{\text {path }-n}^{m}
\end{array}\right]\right\}
$$

target $=\left\{\begin{array}{llll}V_{\text {TOMO-AVR-1 }} & V_{\text {TOMO }-A V R-2} & \ldots & V_{\text {TOMO }}-A V R-m\end{array}\right\}$

Artifical Neural Network (ANN)

ANN is a learning machine method consists of unit computation (neuron) and the configuration of the 
connection between neurons (called architecture) [33]. Given a set of input and the specific of a target, ANN could be trained to calculate a mapping function in the training phase. The capability to calculate generalization of mapping functions between input and target (see Eq. 9) is essential to keep accuracy in the measurement phase.

In this work, further evaluation of performance comparison between ANN with feedforward backpropagation and cascade correlation performed. The illustration of the architecture of both customized ANN to support the proposed scheme of hybrid USM-Tomo shown in Figure 8.

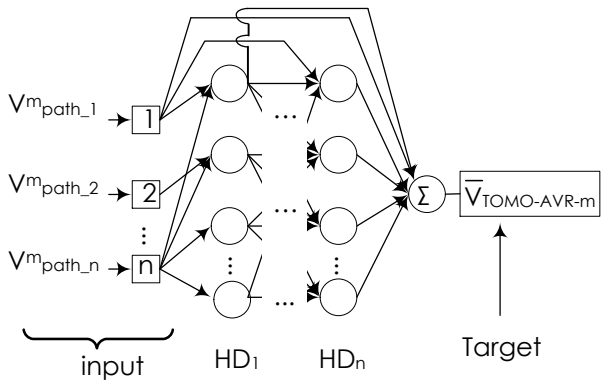

(a)

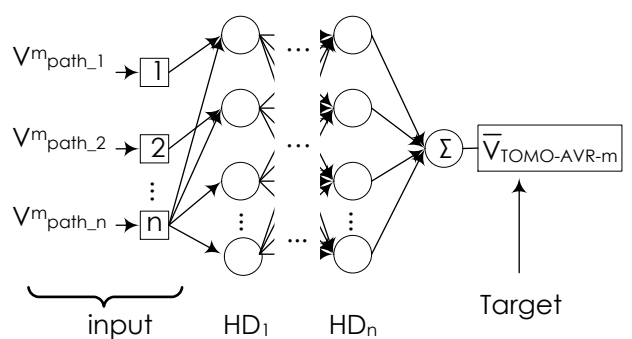

(b)

HDx $=$ Hidden layer $x^{\text {th }}$ with activation function

Figure 8 Architecture of (a) cascade correlation and (b) back-propagation

\section{Support Vector Regression (SVR)}

Another machine learning could also be used to determine the adaptive weighting in USM is SVR [10], [25]. Basically, in SVR mapping between input and output can be viewed as a linear regression data. For multipath USM, the data set of $\left\{\mathbf{V} \mathbf{m}_{\text {path-1 }}, \mathbf{V} \mathbf{m}_{\text {path }}\right.$ $\left.2, \ldots . . ., V \mathbf{m}_{\text {path-n }}\right\}$ transformed into a new set value $\left\{\mathbf{X}_{1}, \mathbf{X}_{2}, \ldots \ldots . \mathbf{X}_{\mathbf{s}}\right\}$, that denoted by $\mathbf{K}\left(\mathbf{X}_{\mathbf{s}}, \mathbf{V}^{\left.\mathbf{m}_{\text {path-n}}\right)}\right)$ using Kernel function $(\mathbf{K})$, in order to optimize the learning process in SVR [34]. The illustration of SVR architecture used for hybrid USM-Tomo shown in Figure 9.

On learning process, a new set value $\mathbf{X}_{\mathbf{s}}$ is given a specific set of weight ( $\mathbf{W}_{\mathbf{N}}$ ) as an effort to be mapped into the target. In SVR, the range of generalization of functions can be set up using the proper value of error loss function $(\boldsymbol{\varepsilon})$. To guarantee the learning process using SVR can obtain a solution on global optimal, regularization parameter $\mathbf{C}$ has to be set to get the optimization algorithm.

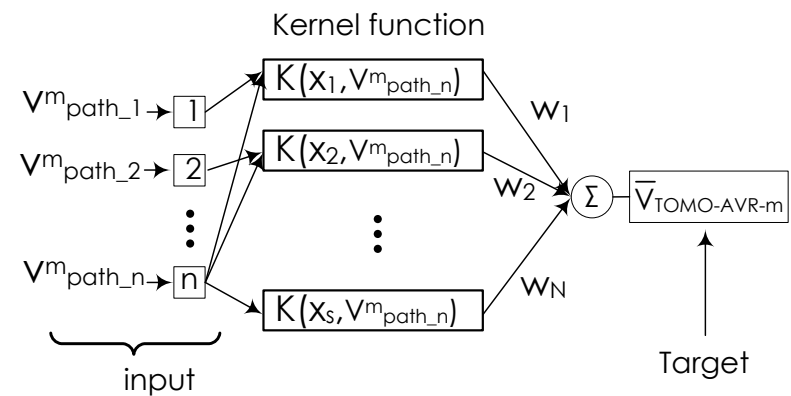

Figure 9 SVR Architecture

\subsection{Numerical Model for Generating Asymmetric Flow Profile}

Salami's model is one of mathematic model that can be used to generate asymmetric flow profiles. Previous work also used Salami's model for researches in related to asymmetric flow profile and measurement [18], [19], [21], [26], [28], [38]. Based on Salami [36], the mathematic model of velocity at each point in crosssection pipe $V\left(r, \theta, k_{1}, k_{2}\right)$ can be a generalization with

$V\left(r, \theta, k_{1}, k_{2}\right)=(1-r)^{k_{1}}+G(1-r)^{k_{2}} F(\theta)$

Where $\mathbf{r}, \boldsymbol{\theta}$ is a pipe radius and angle in polar coordinate. The pattern of the velocity profile is determined parameter $\mathbf{k}_{1}, \mathbf{k}_{2}, \mathbf{G}$ as a constant value and $F(\theta)$ as a specific function. In [36], the 23 sets parameters of Eq-10 is defined, resulting different 23 profile of $\mathbf{V}\left(\mathbf{r}, \mathbf{q}, \mathbf{k}_{1}, \mathbf{k}_{2}\right)$. In each set parameters that defined in Eq 10, the profile is namely profile P1, P2,... and P23. Two examples of parameter that used to generated asymmetry flow profile, i.e.

- $\quad$ parameters of profile P2: $k_{1}=1 / 9, k_{2}=2, G=6,7501 \mathrm{r}$ and $F(\theta)=e^{-0.5 \theta} \sin \theta$, and

- $\quad$ parameter of profile P12: $\mathrm{k}_{1}=1 / 7, \mathrm{k}_{2}=1 / 9, \mathrm{G}=\mathrm{r}$ and $F(\theta)=e^{(0.05 \pi-0.2 \theta)} \sin \theta$.

In this work, a numerical model of asymmetry flow profile used for determining the optimal number of transducers of USM-Tomo, optimal acoustic parallel path USM, and data sets for evaluating performance of ANN and Support Vector Machine (SVM) in USMTomo.

\subsection{Simulation for Optimal Number of Transducers in USM-Tomo}

To determine an optimal number of transducers in USM-Tomo, two asymmetry flow profile based on Eq10 generated by parameter in [36]. P12 represents an asymmetric velocity profile with 1 peak at each point in the cross-section pipe. Then, some points in the boundary of the pipe are used to determine Vpath-n, 
where the number of $\mathrm{n}$ is related to some points. Using $\mathrm{n}$ points configuration, the distance between the point on the boundary of pipe is $2 \pi$ dan pipe radius $/ \mathbf{n}$. Figure 10 shown the example of the configuration using 12 points, that represented as transducers model.

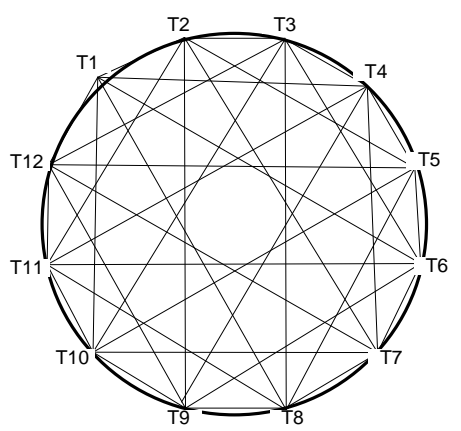

Figure 10 USM-TOMO path configuration using 12 points that represented transducers model

In the simulation, the point numbers are variation between 10, 12, 14 and 20 .

\subsection{Simulation for Optimal Number of Parallel Acoustic Paths in USM}

The number of the acoustic path in USM could influence temporal resolution and measurement accuracy. In order to get the optimal number of path, numerical simulation is performed by selecting a specific acoustic path illustrated in Figure 11. Black lines indicate the used path while the red lines indicate the un-used path line.

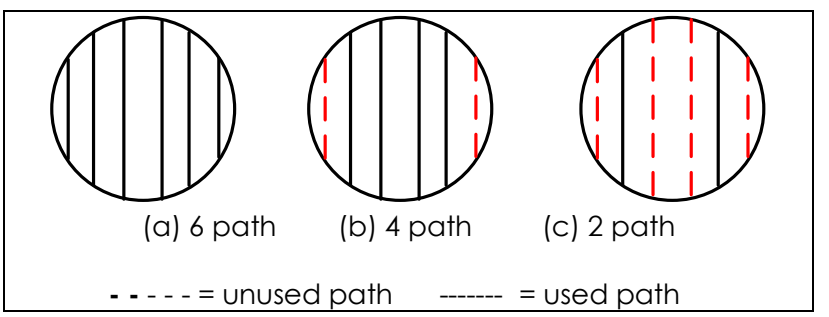

\subsubsection{Data Simulation for Training and Measurement Phase}

The set of data input represents a simulation of velocity in each path of USM and target as the average velocity of the cross-section flow profile was managed. Data sets of velocity on $\mathbf{n}$ path of USM (input) and $V_{\text {avr }}$ (target) which were generated from models of asymmetric flow profile [36].

The data sets split into three groups, where the ratio of the data set is

- Group I = $73 \%$ training phase and $27 \%$ measurement phase

- Group $\|=63 \%$ training phase and $37 \%$ measurement phase

- Group $\| I=53 \%$ training phase and $47 \%$ measurement phase

Three groups of data sets used as data set for evaluation of ANN and SVR in order the determine mapping function $\mathbf{F ( . ) . ~ T h e ~ p e r f o r m a n c e ~ o f ~ b o t h ~}$ machine learning methods was done using an objective criterion that defined as

$$
R M S E=\frac{1}{N} \sqrt{\sum_{1}^{N}\left(V_{\text {avr }}-\bar{v}_{\text {theoretic }}\right)^{2}}
$$

Where $\overline{\mathbf{V}_{\text {theoretic }}}$ is the average velocity of Salami's profile, $\mathbf{N}$ denotes the number of testing data. According to AGA-9, the value of RMSE is less than $1 \%$. Its RMSE value is the maximum allowable total error for an 8-inch pipe is choose for performance evaluation.

\subsection{Experiment Setup for Generating Asymmetric Flow Profile}

USM-Tomo with 12 transducers are made using the principle of dual-transducers (see Figure 12). All transducers are installed using the protrusion technique [15], [16] and form 450 angle relative to the pipe wall, to optimize wave propagation to all receivers. Ultrasonic air transducers with $40 \mathrm{kHz}$ are used in the experiment setup [29].

Figure 11 Different number and position of parallel paths

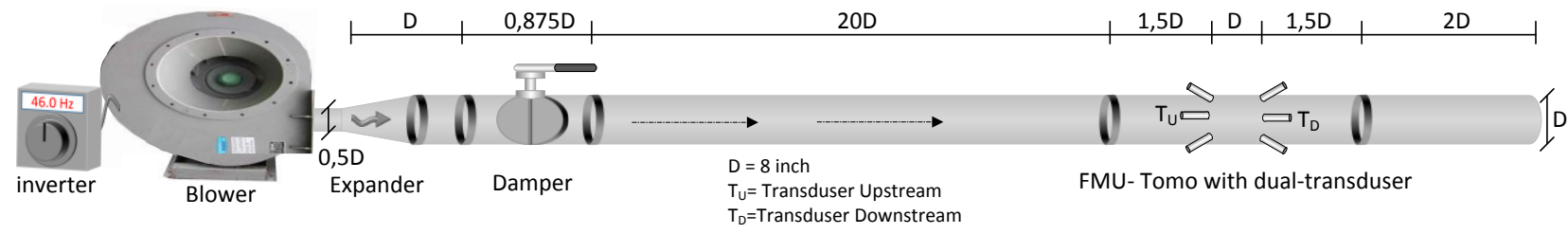

Figure 12 Experiment set-up 
In order to generate asymmetric flow profiles with variable air velocity, air blower (maximum speed 11.3 $\mathrm{m} / \mathrm{s}$ in outlet) with variable speed driver and butterfly damper are used. The damper blade angle can be altered manually so that the shape of the asymmetric profiles. The expander serves to connect 4-inch blower outlet to 8 -inch damper. The meter run pipe of USMTomo is 20D upstream and 2D downstream. The experiment begins by setting the angle of the damper position. The blade angle varies from $0^{0}$ to $64.3^{\circ}$ in steps of 12.90 . Visualization of the blade position for different blade angles are shown in Figure 13 a-b. The pattern of asymmetric flow profiles is also confirmation using hot-wire anemometer. The hot-wire probes are placed in 25 points in pipe's cross-section, as shown in Figure 13-c.

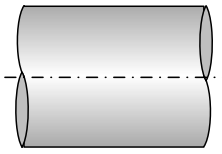

(a) $\alpha=0^{0}$

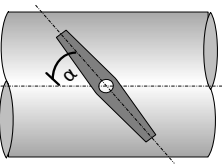

(b) $\alpha=25.70$

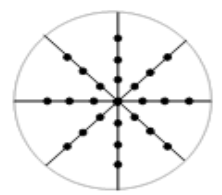

(c)
Figure 13 Visualization of damper opening angle (a) $\alpha=0^{0}$ and (b) $\alpha=25,70$. (c) twenty-five point of velocity measurement using hot wire

An inverter is used to control the speed of blower by varying the frequency of supply from $25-50 \mathrm{~Hz}$. Thus, there are 26 variations of air-speed for each damper position angle and 156 experimental data.

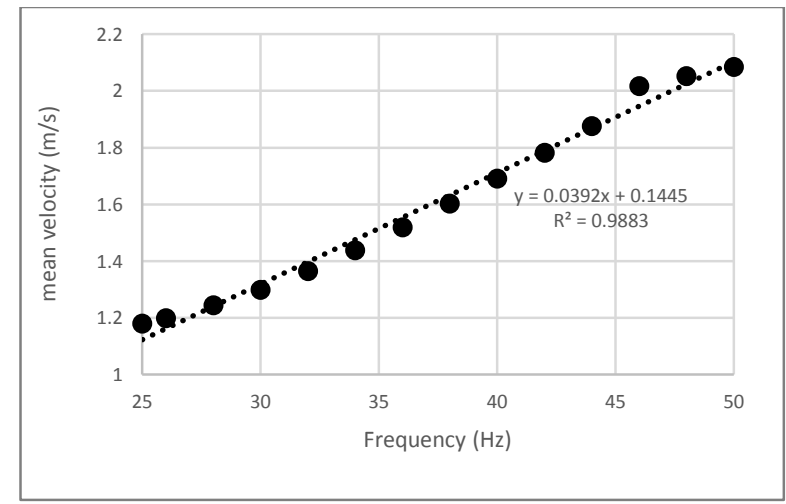

Figure 14 The frequency inverter in relation to average air velocity when the damper blade angle is $38.6^{\circ}$

The example of the relationship between the frequency inverter and average air velocity (blade angle $38.6^{\circ}$ ) shown in Figure 14. The experimental data show that the lowest air-speed is $0.8 \mathrm{~m} / \mathrm{s}$ when the frequency of the supply is $25 \mathrm{~Hz}$ and damper opening angle is $64.3^{\circ}$, the highest is $3.9 \mathrm{~m} / \mathrm{s}$ with $50 \mathrm{~Hz}$ and without damper.

\subsection{RESULTS AND DISCUSSION}

\subsection{Optimal Number of Transducers in USM-Tomo}

Asymmetric Flow Profile based on Model P2 and P12 generated, then an average of flow velocity model P2 and $\mathrm{P} 12$ are represented in Uavr. Then, USM-TOMO with number of transducer model 10, 12, 14 and 20 are used to reconstructed flow profile both models. Then an average of flow velocity resulting from USM-TOMO represented in V'омо-AVR, and performance of reconstructed results based on error (err) that formulated in Eq. 11.

$$
e r r=\left|\frac{U_{a v r}-V_{T O M O-A V R}}{V_{\text {TOMO-AVR }}}\right|
$$

The results are summarized in Table 1. By increasing the number of transducers up to 20 , the minimum error can be obtained on the use of 12 transducers. Based on simulation results, we choose the configuration of 12 transducers in USM-TOMO, as the parameter used in the experiment.

Table 1 Reconstruction of asymmetric flow profile of P2 and P12 with number of transducer models $10,12,14$ and 20

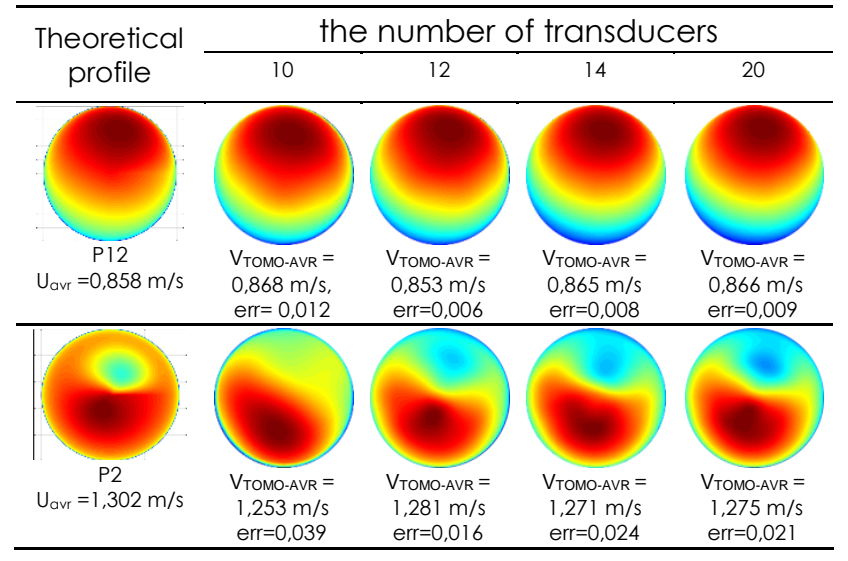


Table 2 proposed architectures of back-propagation and cascade correlation

\begin{tabular}{|c|c|c|c|c|c|c|c|c|}
\hline Network & & & & & back-propa & yation & & \\
\hline Name & $111^{*}$ & 112 & 121 & 122 & 123 & 131 & 131 & 133 \\
\hline $\begin{array}{l}\text { Hidden layer } \\
\rightarrow \text { neuron }\end{array}$ & $1 \rightarrow[25]$ & $1 \rightarrow[25]$ & $2 \rightarrow\left[\begin{array}{lll}25 & 25\end{array}\right]$ & $2 \rightarrow\left[\begin{array}{lll}25 & 25\end{array}\right]$ & $2 \rightarrow\left[\begin{array}{ll}25 & 25\end{array}\right]$ & $3 \rightarrow\left[\begin{array}{lll}25 & 25 & 25\end{array}\right]$ & $3 \rightarrow\left[\begin{array}{lll}25 & 25 & 25\end{array}\right]$ & $3 \rightarrow\left[\begin{array}{lll}25 & 25 & 25\end{array}\right]$ \\
\hline $\begin{array}{l}\text { activation } \\
\text { function }\end{array}$ & Tansig & Logsig & $\begin{array}{l}\text { 1. Tansig } \\
\text { 2. Tansig }\end{array}$ & $\begin{array}{l}\text { 1. Logsig } \\
\text { 2. Logsig }\end{array}$ & $\begin{array}{l}\text { 1. Tansig } \\
\text { 2. Logsig }\end{array}$ & $\begin{array}{ll}\text { 1. } & \text { Tansig } \\
\text { 2. } & \text { Tansig } \\
\text { 3. } & \text { Tansig } \\
\end{array}$ & $\begin{array}{ll}\text { 1. } & \text { Logsig } \\
2 . & \text { Logsig } \\
\text { 3. } & \text { Logsig } \\
\end{array}$ & $\begin{array}{ll}\text { 1. } & \text { Tansig } \\
\text { 2. } & \text { Logsig } \\
\text { 3. } & \text { Purelin } \\
\end{array}$ \\
\hline Learning rate & & & & & $1.10^{-5} ; \ldots$ & 0.1 & & \\
\hline Network & & & & & ascade cor & lations & & \\
\hline Name & $211^{*}$ & 212 & 221 & 222 & 223 & 231 & 231 & 233 \\
\hline $\begin{array}{l}\text { Hidden layer } \\
\rightarrow \text { neuron }\end{array}$ & $1 \rightarrow[25]$ & $1 \rightarrow[25]$ & $2 \rightarrow\left[\begin{array}{ll}25 & 25\end{array}\right]$ & $2 \rightarrow\left[\begin{array}{ll}25 & 25\end{array}\right]$ & $2 \rightarrow\left[\begin{array}{ll}25 & 25\end{array}\right]$ & $3 \rightarrow\left[\begin{array}{llll}25 & 25 & 25\end{array}\right]$ & $3 \rightarrow\left[\begin{array}{llll}25 & 25 & 25\end{array}\right]$ & $3 \rightarrow\left[\begin{array}{llll}25 & 25 & 25\end{array}\right]$ \\
\hline $\begin{array}{l}\text { activation } \\
\text { function }\end{array}$ & Tansig & Logsig & $\begin{array}{l}\text { 1. Tansig } \\
\text { 2. Tansig }\end{array}$ & $\begin{array}{l}\text { 1. Logsig } \\
\text { 2. Logsig }\end{array}$ & $\begin{array}{l}\text { 1. Tansig } \\
\text { 2. Logsig }\end{array}$ & $\begin{array}{ll}\text { 1. } & \text { Tansig } \\
\text { 2. } & \text { Tansig } \\
\text { 3. } & \text { Tansig } \\
\end{array}$ & $\begin{array}{ll}\text { 1. } & \text { Logsig } \\
\text { 2. } & \text { Logsig } \\
\text { 3. } & \text { Logsig }\end{array}$ & $\begin{array}{ll}\text { 1. } & \text { Tansig } \\
\text { 2. } & \text { Logsig } \\
\text { 3. } & \text { Purelin }\end{array}$ \\
\hline Learning rate & & & & & $1.10^{-5} ; \ldots$ & .1 & & \\
\hline
\end{tabular}

Table 3 RMSE of 16 ANN architectures when using 6 parallel path

\begin{tabular}{|c|c|c|c|c|c|c|c|c|c|c|c|c|c|c|c|c|c|}
\hline Structure & & & & ack-prc & dagatio & & & & & & & scade & prelati & & & & \\
\hline Name & 111 & 112 & 121 & 122 & 123 & 131 & 132 & 133 & 211 & 212 & 221 & 222 & 223 & 231 & 232 & 233 & \\
\hline RMSE & 0.055 & 0.099 & 0.063 & 0.117 & 0.044 & 0.067 & 0.093 & 0.050 & 0.026 & 0.033 & 0.034 & 0.135 & 0.022 & 0.078 & 0.060 & 0.007 & 0.009 \\
\hline
\end{tabular}

3.2. Evaluation Performances ANN and SVM for Support Parallel Acoustic Paths in USM

\subsubsection{ANN Performance}

The performance of ANN-based on backpropagation (BP) and cascade correlation (CC) are evaluated. Eight combinations of architectures of BP and $\mathrm{CC}$ tested with variations on the number of hidden layers (from 1 to 3), activation functions and learning rate (LR) (See Table 2).

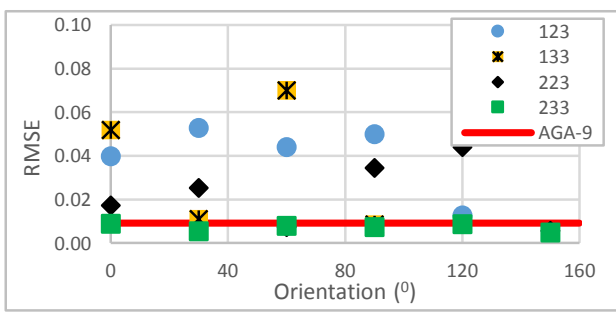

(a)

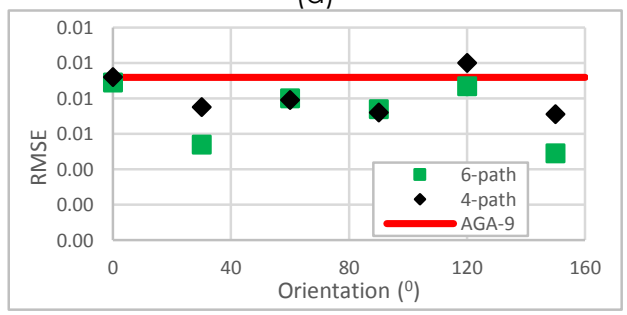

(b)

Figure 11(a) Performance of four ANN structures with 6 parallel path and (b) ANN-233 performance with training and testing ratio of 73:27

The architectures of BP and $\mathrm{CC}$ has 25 neurons in each hidden layer. Each combination of BP and CC used to predict $V_{\text {avr }}$ using 6 parallel paths. The RMSE (see Eq. 10) of each ANN architectures shown in Table 3 and Figure 11 a.
The results shown that the minimum RMSE in 233-CC architecture. Next, the performance of 233-CC architecture was tested using 4, and 6 parallel paths and data sets that label as Group I, II and III. The minimum RMSE could be achieved for data sets that label as Group I and 6 parallel paths (see Figure $11 \mathrm{~b}$ ).

\subsubsection{SVR Performance}

In order to obtain the best SVR architecture, a combination of SVR parameters, i.e. kernel functions $(K)$, error loss function $(\varepsilon)$ and regularization parameter (C) are attempted. The parameters that used to optimize SVR architecture represents in Table 4. The RMSE of each SVR architecture was tested using 6 parallel paths. Based on the evaluation, SVR with a kernel with a cubic polynomial function produce RMSE satisfy AGA-9 (see Figure 12a).

Table 4 SVR proposed architectures

\begin{tabular}{cccc}
\hline Name & SVR-Linr & SVR-Poly & SVR-RBF \\
\hline $\begin{array}{c}\text { Kernel } \\
\text { Function }\end{array}$ & - & Cubic Poly. & RBF \\
\hline Epsilon $(\varepsilon)$ & & $0.1, \ldots, 1.10^{-5}$ & \\
\hline
\end{tabular}

Next, the performance of SVR -Poly was tested using 4, and 6 parallel paths and data sets that label as Group I. The minimum RMSE satisfy AGA-9 could be achieved for $\varepsilon$ is $1.10^{-3}$ and 6 parallel paths (see Figure 12b). 


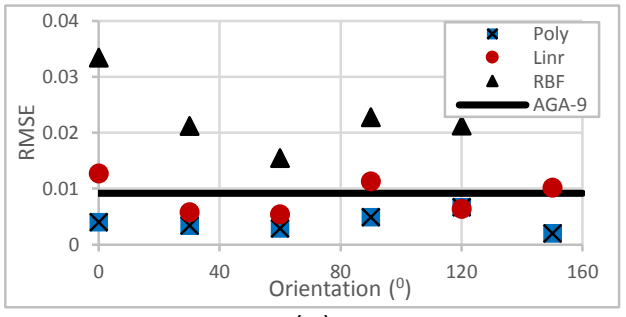

(a)

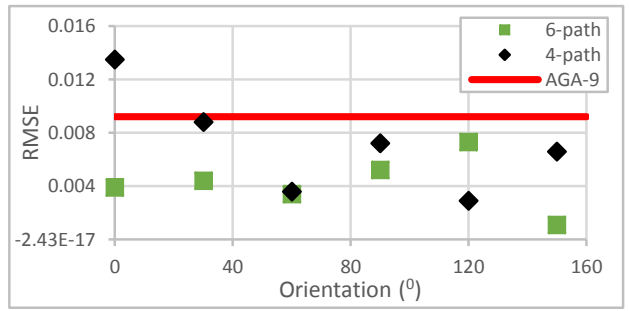

(b)

Figure 12 (a)Performance of the 3 proposed architectures of SVR when using 6 parallel paths (b) SVR-Poly performance with data sets that label as Group I

\subsubsection{Noise Effect on ANN and SVR}

Further evaluation of performance ANN-233 and SVRPoly tested using Salami's model of flow profile with injecting Gaussian noise. A noisy flow profile sets with 4 different signal to noise ratio (SNR): 25, 30, 35, $40 \mathrm{~dB}$. Example of noisy flow profile are shown in Table 5.

Table 5 Salami's profile with Gaussian noise

\begin{tabular}{|c|c|c|c|c|}
\hline \multirow{2}{*}{$\begin{array}{c}\text { Salami's } \\
\text { profiles }\end{array}$} & \multicolumn{4}{|c|}{ SNR } \\
\hline & $25 \mathrm{~dB}$ & $30 \mathrm{~dB}$ & $35 \mathrm{~dB}$ & $40 \mathrm{~dB}$ \\
\hline
\end{tabular}

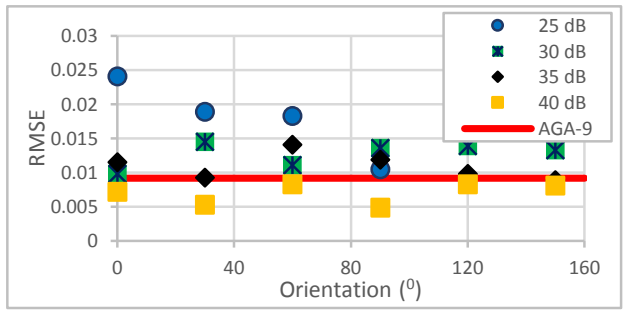

(a)

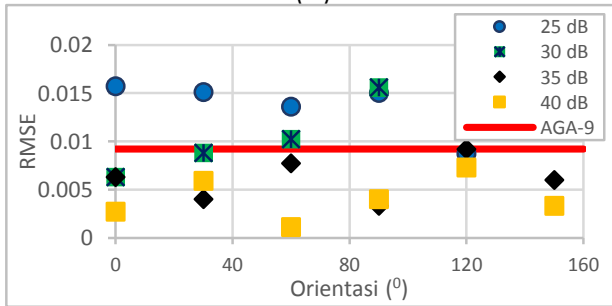

(b)

Figure 13 Effect of A noisy flow profile sets with 4 different SNR on (a) ANN-233 and (b) SVR-Poly with 6 paths
On the previous evaluation, ANN-233 and SVR-Poly produce the RMSE less than maximum allowable percentage error of AGA-9, for data sets that label as Group-l and using 6 parallel paths. In this step, A noisy flow profile sets with 4 different SNR was used as input data for ANN-233 and SVR-Poly. The ANN-233 could be maintained acceptable RMSE if SNR of the data sets bigger than $40 \mathrm{~dB}$. The robustness due to noisy data of SVR-Poly was slightly better than ANN-233 because SVR-Poly can produce acceptable RMSE, although the SNR of the data sets more than $35 \mathrm{~dB}$. The summary of these evaluation step shown in Figure 13.

\subsection{USM-TOMO Performances in Experiment}

\subsubsection{Initial Test of US transducer for USM-TOMO experiment}

Initial test procedure was done to ensure that the USMTOMO operates correctly in the experiment. In the first test, measurement the value of ultrasonic travelling time of upstream (tu) and downstream ( $\left.t_{R}\right)$ for each acoustic path are performed without airflow in the pipe. In this test, the deviation map of $t_{u}$ and $t_{R}$ from ideal condition was determined to compensation tu and $t_{R}$ in the airflow experiment [6].

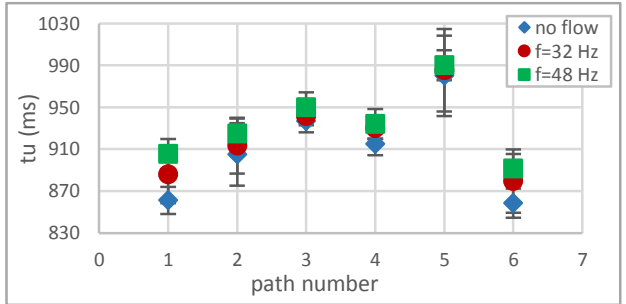

(a)

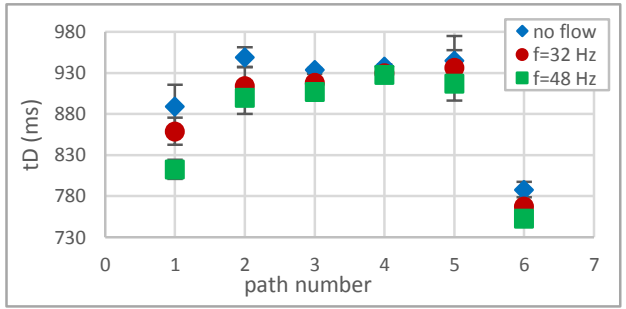

(b)

Figure 14 Direct ultrasonic traveling time to (a) tu and (b) to with no damper and $120^{\circ}$ orientation angle

Second test, the airflow in the pipe is controlled with a variable speed blower $(f=32 \mathrm{~Hz}$ and $f=48 \mathrm{~Hz})$, and the value of tu and $t_{R}$ are measured with setup $120^{\circ}$ of paths orientation angle. The results shown in Figure 14. The proportional value of tu and $t_{R}$ can be achieved in our proposed experiment setup. With increasing speed blower, the tu is getting smaller; on the contrary, the $t_{R}$ becomes larger. 


\subsubsection{Initial Evaluation of Asymmetric Flow Profile between USM-TOMO and Anemometer}

Comparison of particular flow velocity data from USMTomo and hot-wire anemometer shown in Figure 15a with $50 \mathrm{~Hz}$ frequency inverter and 38.60 damper blade angle setup. The graph reveals that there is an offset even though the distribution of flow velocity has an almost identical pattern. For additional information, none of the hot-wire probe coincide directly with the USM-Tomo acoustic paths.

The example of the reconstructed profile using USM-TOMO in the setting $44 \mathrm{~Hz}$ inverter and damper blade angle $0^{\circ}$ and $38.6^{\circ}$ are demonstrated in Table 6. Comparison $\mathbf{V}_{\text {avr }}$ that determined using USM-TOMO and hot wire anemometer also shown in Table 6. In general, the error ranges of USM-Tomo are from $0 \%$ to $14 \%$ and $4 \%$ for the mean error if the hot wire anemometer used as reference.

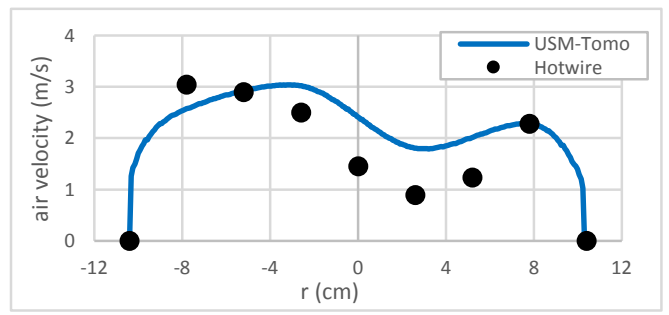

(a)

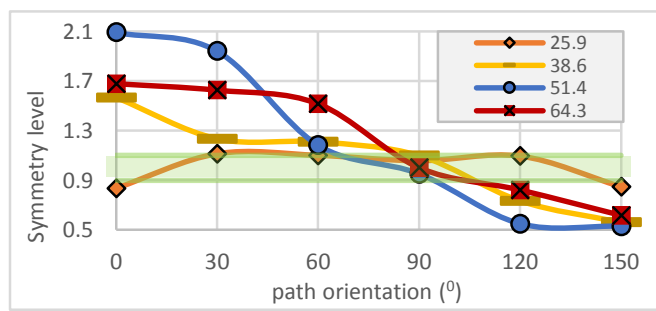

(b)

Figure 15 (a) Comparison of particular flow velocity data from USM-Tomo and hot-wire at $y=-x$ and $38.6^{\circ}$ damper blade angle (b) Asymmetrical level of flow profile for four different blade angles

The asymmetrical level of flow profile (AR) is calculated using Eq. (11). The RR value equal 1 represents for symmetry of flow profile. The USM-TOMO scheme can maintain its accuracy when the AR lies between 0.85 and 1.15 (see Figure 15-b) [37]. The plot of AR value in different damper blade angles shown in Figure 15b.

$$
A R=\frac{v_{1}+v_{2}+v_{3}}{v_{4}+v_{5}+v_{6}}
$$

Figure $1-5 b$ shows that when the damper blade higher than 38.60, the flow profiles become very asymmetrical.
Table 6 The flow profile of $44 \mathrm{~Hz}$ inverter when damper angle is varied

\begin{tabular}{|c|c|c|c|c|}
\hline \multirow{2}{*}{$\alpha$} & \multicolumn{3}{|c|}{ USM-Tomo } & Hot wire \\
\hline & Profiles 2D & Profiles 3D & $V_{\text {avr }}(\mathrm{m} / \mathrm{s})$ & $\mathrm{V}_{\mathrm{avr}}(\mathrm{m} / \mathrm{s})$ \\
\hline $0^{0}$ & & & $V_{a v r}=3,6$ & $V_{a v r}=3,1$ \\
\hline $38.6^{\circ}$ & & & $V_{\mathrm{avr}}=2,0 \mathrm{~m} /$ & $\mathrm{V}_{\mathrm{avr}}=2,0$ \\
\hline
\end{tabular}

\subsubsection{Performance of hybrid USM-Tomo with ANN and SVR}

In the training phase of the hybrid USM-Tomo, a mapping between the set of velocity on six parallel path of USM-Tomo (input) and $\mathrm{V}_{\text {TOMO-AVR }}$ (target) are performed using ANN-233 and SVR-Poly as shown in Figure 6 . In the simulation, $73 \%$ of experimental data used as training sets and the rest for testing sets. The experimental results show that the shape of flow profiles from different damper blade angles are different from each other, as shown in Table 6. Meanwhile, the pattern of flow profiles is almost equal for each damper blade angles, as displayed in Table 7. Based on those conditions, hybrid USM-Tomo with ANN-233 or SVR-Poly are trained and tested for every blade angle.

Table 7 The profile form when air velocities are varied

\begin{tabular}{|c|c|c|c|}
\hline \multirow[t]{2}{*}{ Damper } & \multicolumn{3}{|c|}{$(\mathrm{Hz})$} \\
\hline & $2,0 / 25$ & $3,2 / 40$ & $3,6 / 44$ \\
\hline $0^{\circ}$ & & & \\
\hline
\end{tabular}

Asymmetrical level in Figure $18 \mathrm{~b}$ reveals that velocity profile experimental data can be grouped into two conditions; symmetric and asymmetric flow. Comparison of AGA-9, hybrid USM-Tomo with ANN-233 or SVR-Poly performances for symmetric profiles are shown in Figure 19a and the asymmetric in Figure 19b. The figures show the predicted values obtained by ANN-233 and SVR-Poly are almost close to the target value of USM-Tomo. The RMSE of ANN-233 and SVRpoly are far lower than AGA-9, which means the presence of small error predictions. Moreover, if the two methods are compared, the RMSE of SVR-Poly is generally smaller than ANN-233 method. It indicates that the mapping capability of SVR-Poly is better than ANN-233. Both algorithms work effectively in mapping various asymmetric and symmetric flow profiles for both numerical and experimental data. This technique is more effective than reference [23] in 
which the ANN architecture has to be redesigned for every profile form.

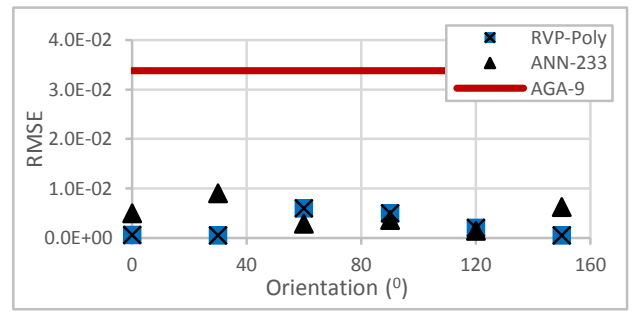

(a)

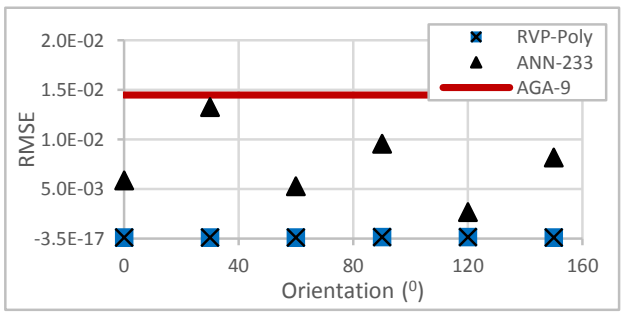

(b)

Figure 19 RMSE of ANN-233 and SVR-Poly with damper angle (a) $0^{\circ}$ and (b) $64.3^{\circ}$

Regarding the issue of temporal resolution of USMTomo, by using the hybrid USM-Tomo and dualtransducers technique for data collection strategy, the sampling period becomes shorter from about \pm $160 \mathrm{~ms}$ [28] to only $\pm 7 \mathrm{~ms}$. Moreover, the computation time of the tomographic method is around $0.82 \mathrm{~s}$, but for hybrid USM-Tomo using SVR-Poly or ANN-233 are around 0.01-0.03s or 0.05-0.09s, respectively. Thus, the proposed method is possible to be an alternative solution to obtain USM system which has high temporal resolution, good accuracy for symmetric and asymmetric flow, and easy adjustment for different circumstances.

\subsection{CONCLUSION}

The alternative scheme of USM for asymmetric profiles was presented. The novel scheme on the training phase is a strategy to determine the mapping function between the flow velocities on an individual acoustic path and the average flow velocity on a cross-section of a pipe based on the hybrid USM-Tomo. This scheme has the potential to be used industrial flow meter because it is based only on time flight ultrasonic for generating input and target data in the training phase.

The heart of this scheme that is proven useful is the programmable acoustic path configuration that could set on the path configuration mode between USM parallel path and tomography. The measurement of time of flight on each acoustic path can be speed up by using dual-transducers technique. Based on performance evaluation on data simulation and experiment, ANN cascade correlation and SVR polynomial with 6 parallel paths can keep USM error below $1 \%$ compared to tomography. Moreover, the measurement time is only $\pm 7 \mathrm{~ms}$ and the computational time of the hybrid USMTomo with SVR polynomial and ANN cascade correlation are 0.01-0.03s and 0.05-0.09s, respectively. Thus, the hybrid USM-Tomo is potential to be implemented in industrial application.

\section{References}

[1] L. Zhang, L. $X U$, and L. Rai. 2018. High-precision Ultrasonic Flowmeter for Mining Applications based on Velocity-area. Telkomnika. 16(1): 84-93.

[2] V. Leontidis, C. Cuvier, G. Caignaert, P. Dupont, O. Roussette, S. Fammery, P. Nivet3, and A. Dazin. 2018. Experimental Validation of an Ultrasonic Flowmeter for Unsteady Flows Application. Meas. Sci. Technol. IOP Publishing. 29 (4): 045303.

[3] A. Hallanger, C. Saetre, and K.-E. Froysa. 2018. Flow Profile Effects Due to Pipe Geometry in an Export Gas Metering Station-Analysis by CFD Simulations. Flow Meas. Instrum. 61: 56-65.

[4] Q. Chen and J. Wu. 2014. Research on the Inherent Error of Ultrasonic Flowmeter in Non-ideal Hydrogen Flow Fields. Int. J. Hydrogen Energy. 39(1 1): 6104-6110.

[5] C. Wang, T. Meng, H. Hu, and L. Zhang. 2012. Accuracy of the Ultrasonic Flow Meter Used in the Hydroturbine Intake Penstock of the Three Gorges Power Station. Flow Meas. Instrum. 25: 32-39.

[6] AGA Report No. 9. 2007. Measurment of Gas by Multipath Ultrasonic Meters. Second Editon. American Gas Association, 400 North Capitol Street NW, Washiton

[7] H. Zhao, L. Peng, S. A. Stephane, H. Ishikawa, K. Shimizu, and M. Takamoto. 2014. CFD Aided Investigation of Multipath Ultrasonic Complex Flow Profile. IEEE Sens. J. 14(3): 897-907.

[8] G. J. Brown and B. W. Griffith. 2013. A New Flow Conditioner for 4-Path Ultrasonic Flowmeters. 24-26.

[9] L. C. Lynnworth and Y. Liu. 2006. Ultrasonic Flowmeters: Half-century Progress Report, 1955-2005. Ultrasonics. 44: e1371-e1378.

[10] H. Zhao, L. Peng, T. Takahashi, and T. Hayashi. 2014. Support Vector Regression-Based Data Integration Method for Multipath Ultrasonic Flowmeter. IEEE Trans. Instrum. Meas. 1-9.

[11] E. Liu, H. Tan, and S. Peng. 2017. A CFD Simulation for The Ultrasonic Flow Meter with a Header. Teh. Vjesn. 6(24): 1797-1801.

[12] L. Zhang, H. Hu, T. Meng, C. Wang, N. Third, R. Road, and C. Dist. 2013. Effect of Flow Disturbance on MultiPath Ultrasonic Flowmeters. IMEKO.

[13] I. Gryshanova, I. Korobko, and P. Pogrebniy. 2016. Increasing of Accuracy of Multipath Ultrasonic Flow Meters by Intelligent Correction. Meas. Autom. Monit. 62(12): $411-416$.

[14] Y.Sun, T. Zhang, and Dandan Zheng. 2018. New Analysis Scheme of Flow-acoustic Coupling for Gas Ultrasonic Flowmeter with Vortex near the Transducer. Sensors. 18(4): 1151.

[15] B. Wang, Y. Cui, W. Liu, and X. Luo. 2013. Study of Transducer Installation Effects on Ultrasonic Flow Metering Using Computational Fluid Dynamics. Adv. Mater. Res. 629: 676-681.

[16] D. Zheng, P. Zhang, and T. XU. 2011. Study of Acoustic Transducer Protrusion and Recess Effects on Ultrasonic 
Flowmeter Measurement by Numerical Simulation. Flow Meas. Instrum. 22(5): 488-493.

[17] G. Chen, G. Liu, B. Zhu, and W. Tan. 2015. 3D Isosceles Triangular Ultrasonic Path of Transit-time Ultrasonic Flowmeter: Theoretical Design and CFD Simulations. 15, 9: 4733-4742.

[18] D. Kurniadi and A. Trisnobudi. 2006. A Multi-path Ultrasonic Transit Time Flow Meter Using a Tomography Method for Gas Flow Velocity Profile Measurement. Part. Part. Syst. Charact. 23(3-4): 330-338.

[19] P. I. Moore, G. J. Brown, and B. P. Stimpson. 2000. Ultrasonic Transit-time Flowmeters Modelled with Theoretical Velocity Profiles: Methodology. Meas. Sci. Technol. 1802-1811.

[20] K. Tawackolian. 2014. Investigation of a Ten-path Ultrasonic Flow Meter for Accurate Feedwater Measurements. Meas. Sci. Technol. 75304.

[21] E. Mandard, D. Kouam, and R. Battault. 2008. Methodology for Developing a High-precision Ultrasound Flow Meter and Fluid Velocity Profile Reconstruction. IEEE Trans. Ultrason. Ferroelectr. Freq. Control. 55(1): 161-172.

[22] H. Zhao, L. Peng, T. Takahashi, and T. Hayashi. 2014. ANN Based Data Integration for Multi-Path Ultrasonic Flowmeter. IEEE Sens. J. 14(2): 362-370.

[23] L. Hu, L. Qin, K. Mao, W. Chen, and X. Fu. 2016. Optimization of Neural Network by Genetic Algorithm for Flowrate Determination in Multipath Ultrasonic Gas Flowmeter. 16(5): 1158-1167.

[24] L. Qin, L. Hu, K. Mao, W. Chen, and X. Fu. 2016. Application of Extreme Learning Machine to Gas Flow Measurement with Multipath Acoustic Transducers. Flow Meas. Instrum. 49: 31-39.

[25] L. Qin, L. Hu, K. Mao, W. Chen, and X. Fu. 2016. Flow Profile Identification with Multipath Transducers. Flow Meas. Instrum. 52(October): 148-156.

[26] D. Zheng, D. Zhao, and J. Mei, 2015. Improved Numerical Integration Method for Flowrate of Ultrasonic Flowmeter Based on Gauss Quadrature for Non-ideal Flow Fields. Flow Meas. Instrum. 41: 28-35.
[27] X. Tang, X. Xie, B. Fan, S. Member, and Y. Sun. 2018. A Fault-Tolerant Flow Measuring Method Based on PSOSVM With Transit-Time Multipath Ultrasonic Gas Flowmeters. IEEE Trans. Instrum. Meas. 67(5): 992-1005.

[28] J. Liu, B. Wang, Y. Cui, and H. Wang. 2015. Ultrasonic Tomographic Velocimeter for Visualization of Axial Flow Fields in Pipes. Flow Meas. Instrum. 41: 57-66.

[29] C. L. Goh, A. R. Ruzairi, F. R. Hafiz, and Z. C. Tee. 2017. Ultrasonic Tomography System for Flow Monitoring: A Review. IEEE Sens. J. 17(17): 5382-5390.

[30] K. Amri, L. F. Wiranata, S. Suprijanto, and D. Kurniadi. 2015. Fluid Flow Velocity Measurement Using DualUltrasonic Transducer by Means of Simultaneously Transit Time Method. ICICI-BME Conference 2015. 1-4.

[31] A. C. Kak and M. Slaney. 2001. Principles of Computerized Tomographic Imaging. IEEE Press.

[32] S. Kv and B. K. Roy. 2012. An Intelligent Flow Measurement Technique using Ultrasonic Flow Meter with Optimized Neural Network. International Journal of Control and Automation. 5(4): 185-196.

[33] M. T. Hagan and M. H. Beale. 2014. Neural Network Design. 2 edition. Martin Hagan.

[34] A. J. Smola and B. Scholkopf. 2004. A Tutorial on Support Vector Regression. Stat. Comput. 14: 199-222.

[35] S. Frank, C. Heilmann, and H. E. Siekmann. 1997. Pointvelocity Methods for Flow-rate Measurements in Asymmetric Pipe Flow. Flow Meas Instrum. 7.

[36] L. A. Salami. 1984. Asymmetric Flow Measurement in Circular Pipes. Transactions of the Institute of Measurement and Control. 6(4): 197-206.

[37] G. J. Brown, D. R. Augenstein, and T. Cousins. 2006. An 8-Path Utrasonic Master Meter for Oil Custody Transfers. XVIII IMEKO WORLD Congr.

[38] K. Amri, S. Suprijanto,E. Juliastuti and D. Kurniadi. 2017 Asymmetric Flow Velocity Profile Measurement using Multipath Ultrasonic Meter with Neural Network Technique. 5th International Conference on Instrumentation, Control, and Automation (ICA). 146151. 